\title{
Injection and Mixing of Gas Propellants for Pulse Detonation Propulsion
}

\author{
Dora Elia Musielak* \\ Mechanical and Aerospace Engineering Department \\ University of Texas at Arlington (UTA) \\ Arlington, Texas, USA
}

Detonation has received attention of late because of its role as the primary combustion mechanism in rocket and airbreathing Pulse Detonation Engines (PDEs). However, there are many unresolved issues related to the proper utilization of detonation combustion in these applications. The detonation process has been studied predominantly under the assumption of premixed gases. However, the unsteady nature of PDEs requires relatively small times to inject and mix the propellants prior to each cyclic detonation; and this condition is difficult to achieve experimentally. This paper addresses the injection and mixing processes, and presents new data aimed at supporting the development of practical PDEs. Results are given to illustrate the mixing characteristics of fuel-oxidizer mixtures, modeled with a CFD combustion code.

\section{INTRODUCTION}

A Pulse Detonation Engine (PDE) is a propulsion system which operates on the principle of cyclic detonation combustion. Fuel-air or fuel-oxidizer mixtures are introduced into the PDE's combustion chamber and an appropriate ignition system is used to induce a detonation. The relevant energy produced by the intermittent detonation process is extracted for propulsion. The main advantage of the PDE is that the combustion process itself is very fast, higher pressures and temperatures can be achieved in the detonation chamber, and large power densities can be derived. The main goal for development of PDE propulsion is to increase the detonation frequency of the engine, in order to achieve higher levels of thrust.

Although detonation combustion has been studied for many years, "attempts to utilize detonations for practical propulsion systems are relatively recent. Researchers like Bussing and his team at ASI started with shock-tube experiments for the development of PDEs ${ }^{1}$, and later reported a practical implementation of multi-cycle detonation chambers ${ }^{2}$. Wilson and researchers at UTA have developed single and multi-cycle detonation combustion with various fueloxidizer mixtures ${ }^{3-5}$. Eidelman ${ }^{6}$ has patented PDE concepts and has reported results from experimental and computational detonation combustion. Other groups in the USA and abroad are known to have developed pulsed

\footnotetext{
* Assistant Professor, AIAA Member

Copyright $\odot 1998$ by the autor. Published by the American Institute of Aeronautics and Astronautics, Inc., with permission.
} 
detonation combustion for various applications.

Thus, it appears as if the feasibility of utilizing intermittent detonation combustion has already being demonstrated, however, considerable more work is needed to develop an efficient airbreathing or rocket-type PDE operating at competitive thrust levels. A great deal of work has been done already with single-shock experiments in detonation tubes, but not nearly enough with frequency-controlled or cyclic-detonations. This is because detonating propellant mixtures and controlling the detonations to produce thrust in a propulsion system requires more than knowledge of the detonation process itself. Even though several investigators ${ }^{2,6}$ have reported successful operation of detonation chambers at frequencies over $100 \mathrm{~Hz}$, very little has been reported on the detonation combustion and gas dynamics characteristics of such devices. Experimentally, for example, promoting continuous detonations and extracting the energy for thrust remains a challenge. Concluding statements to characterize successful detonation at high frequencies has been derived mainly from pressure data, and in some cases, detonation velocities have also been measured with questionable results. It is believed that difficulties in attempting to study and characterize the performance of PDEs have been caused by inadequate detonation initiation energy ${ }^{2}$.

Information on the gas dynamics and reactants mixing characteristics inside the various PDEs being developed is lacking. It is well recognized that injection of propellants into the detonation chamber and opening of valves, for example, requires a certain amount of time, and this time is dependent on the frequency of operation of the engine. Most of the PDE performance analysis reported to date is based on the assumption of complete instantaneous mixing, which is impossible to achieve experimentally. Thus, unsatisfactory experimental results in many cases could be attributed to poor fuel/oxidizer mixing.

Results from numerical simulations are also scarce. This is due to the rather slow development of computer codes that can calculate the detonation combustion equations, and model the various coupled processes in a PDE. Unsteady detonation waves are difficult to compute because the chemistry equations are very stiff, and in spite of model simplifications, the results are still inadequate. Steady detonation waves are also computationally stiff, but not nearly as much as unsteady ones, thus, steady detonation waves can and have already been modeled with various codes, using a semiimplicit treatment of the chemical reaction terms in the governing equations. With unsteady detonation waves and high amounts of heat release, the semi-implicit treatment is insufficient and a fully implicit treatment is needed. Unfortunately this cannot be done with many of the available codes. One can still use some of the most advanced combustion computer programs for unsteady detonation waves, but the CFL number must be set very small, which makes computational times prohibitively large. Encouraging results have been obtained by the author in the simulation of shockinduced combustion with a Navier-Stokes code which incorporates a multi-species, multi-equation kinetics model. Predictions of the reaction process in the thin zone of the shock were obtained. It was found that, upon injection, the shock wave created a sudden temperature rise above the ignition temperature of the fuel, resulting in chemical 
reaction. The level of combustion was determined by the production of $\mathrm{H}_{2} \mathrm{O}$ and $\mathrm{OH}$ radicals near the injection zone?

This paper reports work in progress using a computational approach to describe the fluid dynamics coupled to the detonation combustion processes. In this paper, data are presented on the flow field characteristics in detonation chambers, and on the mixing profiles of hydrogen-oxygen mixtures simulating injection schemes relevant to PDEs. This work is aimed to support the study of detonation combustion. Ultimately, knowledge gathered. from this research can be used to predict injection and mixing characteristics of reactants other than hydrogen-oxygen, and that can be useful in the design of PDEs.

\section{PHYSICAL PROCESSES}

There are many processes involved in the flow phenomena associated with a pulse detonation engine. For example, injection and mixing of reactants, ignition of the mixture, initiation/propagation of the detonation wave inside the combustion chamber, expansion of detonation products from the chamber prior to a new cycle, all are processes that affect the performance of a PDE. These processes are interdependent, and their interaction and timing are crucial to engine efficiency ${ }^{6}$. Strategies for PDE performance optimization have included studies of recharge timing, ignition, and nozzle area variation ${ }^{4}$. ${ }^{8}$. However, most PDE studies reported to date assume detonation combustion to occur within a perfect detonation chamber, i.e., one where infinitely fast mixing of the reactants occurs. In practice, the fuel and oxidizer are injected into a detonation chamber as separate streams, with different injection conditions. Mixing is therefore not instantaneous.

In addition to the parameters that govern any combustion process, there are others that are very important for the development of a practical PDE. These parameters include detonation limits, initiation energy, critical chamber diameter, quenching diameters, and thickness of the supporting reaction zone. Knowledge of the wave structure and, thus, chemical reaction rates is needed to quantify and qualify these parameters in the framework of a PDE.

For practical airbreathing PDEs, the detonability of mixtures of hydrocarbon fuels with air is of fundamental importance. The spatial scales for fuel-air detonations and the amounts of energy required to initiate the detonations are often very large. Also, prior to detonation, knowledge is needed of the factors that control the injection and mixing of the reactants, to establish the flowfield characteristics in the detonation chamber, and to ensure uniform distribution of the detonable mixture. This is because the mixture ratio determines the level of detonations that can be achieved, and thus the thrust that can be produced by a PDE.

Mixing different gaseous flows can imply a host of processes and phenomena. When mixing is viewed in the context of chemical reactions and combustion, only fluid species mixed on a molecular scale can contribute to chemical product formation and associated heat release. Mixing can dictate the performance of a PDE, which is believed to relay on the details of the turbulent mixing process to ensure a highly uniform detonable mixture prior to ignition. Thus, mixing studies can provide insight into the fundamental interactions between the 
fluid mechanics and chemical kinetic properties of detonation combustion.

It is estimated that it takes approximately 20 percent of the PDE cycle to inject the propellant gases into the PDE chamber, before they are ignited and detonated. This implies that, for a $100 \mathrm{~Hz}$ PDE, the 20 percent cycle time translates into less than $2 \mathrm{msec}$ available for mixing. To promote rapid mixing and ensure short injection times, several injection approaches have been proposed. It is the objective of this research to provide data to characterize the mixing that is experimentally possible with those injection schemes. First, let us address the processes in the PDE cycle.

\section{ENGINE CYCLE}

A better understanding of the thermodynamics of the PDE cycle is needed, as well as the physical and detonability characteristics of the candidate fuels. Let us begin with a review of the theory of detonation combustion and then proceed to examine the thermodynamic cycle of the PDE.

The Zeldovich-vonNeumann-Doring (ZND) theory, for the structure of the detonation, states that the detonation wave consists of a planar shock moving at the detonation velocity and leaving heated and compressed gas behind it. After an induction period, the chemical reaction starts and, as the reaction progresses, the temperature rises and the density and pressure fall until they reach the Chapman-Jouguet (C-J) values, and then the reaction attains equilibrium ${ }^{9}$. A rarefaction wave then sets in. Thus, behind the C-J shock, energy is generated by thermal reaction. It must be noted here that detonation phenomena is dealt mainly with premixed gases.
The variation of the physical parameters in stoichiometric detonation mixtures was calculated using a chemical equilibrium code $^{10}$ for mixtures of interest to PDEs. Typical results for various detonation mixtures are given in Table 1, where state 1 represents the state of the shock front, state 2 is the state of the gases immediately after the shock, and state 3 is the ChapmanJouguet (C-J) state. As shown, as the gaseous mixture passes from the shock front to the C-J state, its pressure drops about a factor of two, the temperature rises about a factor of two, and the density drops by a factor of three. The sensitivity of the detonation parameters to the reactants initial mixture ratio is illustrated in Fig. 1. It should be noted that the detonation velocity is calculated assuming an infinite tube diameter, therefore, experimental values must be corrected before attempting to match detonation parameters with those given by the theory.

Now, let us relate the above detonation model to the three PDE thermodynamic processes, which approach a modified Adkins cycle. In this model, the detonable mixture is compressed by the shock, heat is added at near-constant-volume, and the gas is expanded to some pressure level by the expansion waves that follow the detonation. The PDE cycle diagram is shown in Fig. 2, where states $1,2,3$ correspond to those in Table 1.

The thrust of a PDE will be derived mainly from the C-J pressure exerted on the thrust wall of the detonation chamber $\left(\mathrm{P}_{2}\right)$, and also from the momentum increase in the exhaust stream. As described by the ZND theory, the pressure rise is provided by the detonation shock, which is mainly dependent on the detonation mixture and the initiation energy. It is important to note that 
the pressure ratios in the compression and expansion processes are not equal, and by definition, these processes are not isentropic. It must also be clear that, based on thermodynamic principles, the entropy change during the PDE thermodynamic cycle is directly proportional to the pressure and density gradients ${ }^{9}$, i.e.,

$$
d s=\left(\frac{1}{\gamma-1}\right) \frac{d P}{T \rho}+\left(\frac{\gamma}{\gamma-1}\right) \rho R d\left(\frac{1}{\rho}\right)
$$

Also, the entropy across the detonation wave itself (state 1-2) can be estimated with the normal shock relation, which is a function of the detonation velocity.

Now, as shown in Table 1, the Mach number following the expansion, $M_{3}$, is much lower than the detonation Mach number, $M_{1}$. However, the sound speed at the exit of the detonation chamber, $a_{3}$, is much larger than that at the inlet, $a_{1}$. For example, for a propane-air detonation process, the sonic velocity of the exhaust gases is three times greater than the sonic velocity of the unburned mixture. Thus, assuming that the Mach number at the exit of the detonation chamber is of the order unity, the exit velocity can be increased with a relatively compact divergent nozzle. Results from a systems study to integrate a nozzle to a detonation chamber will be presented in a separate paper.

The detonability of fuels of interest to PDEs must be assessed in the context of propulsion performance. It is beyond the scope of the present paper to review the literature or to address the issue. However, the interested reader is referred to research with detonation tubes reported in References 12-17.

\section{RESULTS AND DISCUSSION}

This section describes results from two separate studies. One is based on a transient analysis performed to estimate the gaseous propellants injection time. The second is a CFD study to model the injection and mixing of reactants in detonation chambers. This is part of a more comprehensive research program that includes detonation combustion. The computer code being developed calculates chemical reaction of hydrogen-air and hydrogen-oxygen at very high velocities. A new kinetics model for hydrocarbon fuels is being considered ${ }^{18}$.

\subsection{Injection Time}

In the context of a pulse detonation chamber, "filling time" is a misnomer since the detonation chamber is open at one end. Therefore, in this paper the term "injection time" will be used instead. One can estimate the time it will take for a propellant gas to occupy a given chamber volume. This can be done by assuming the pressurized propellant enters the PDE through a choked injector nozzle of a given throat area, and performing a transient analysis. Making use of the ideal gas law, the (unsteady) conservation of mass principle, and assuming isentropic process for the injection process, one obtains a differential equation that can be integrated to obtain the time required for injection of propellants into a combustion chamber of a given volume. The analysis begins by differentiating the perfect gas equation with respect to time:

$$
V \frac{d p}{d t}=m R \frac{d T}{d t}+R T \frac{d m}{d t}
$$

where the mass rate for choked flow is given by: 


$$
\frac{d m}{d t}=-P A\left[\frac{\gamma}{T R}\left(\frac{2}{\gamma+1}\right)^{(\gamma+1) /(r-1)}\right]^{1 / 2}
$$

And, for isentropic flow:

$$
P^{(1-\gamma) / \gamma} T=C
$$

a function which is differentiated with respect to time and rewritten as:

$$
\frac{d T}{d t}=\frac{\gamma-1}{\gamma} \frac{T}{p} \frac{d p}{d t}
$$

Combining equations (1), (2), and (3), it yields:

$$
v \frac{d p}{d t}=m R\left(\frac{\gamma-1 T d p}{\gamma p d t}\right)-R T\left[p A \sqrt{\frac{\gamma}{R T}\left(\frac{2}{\gamma+1}\right)^{(\gamma+1) /(\gamma-1)}}\right]
$$

Let $T=T_{1}\left(p / p_{1}\right)^{(\gamma-1) / \gamma}$, where $T_{1}$ and $P_{1}$ are the initial temperature and pressure of the gas, and rewrite the above equation as follows:

$$
\frac{d p}{d t}=-p^{(\gamma-1) / 2 \gamma}\left[\frac{A \quad \gamma R}{V p_{1}^{(\gamma-1) / 2 \gamma}} \sqrt{\frac{\gamma T_{1}}{R}\left(\frac{2}{\gamma+1}\right)^{(\gamma+1) /(\gamma-1)}}\right]
$$

Separating variables and integrating the above equation results in the following relationship for the injection time, $t$ :

$$
t=\frac{2 V\left[1-\left(\frac{P_{1}}{P_{2}}\right)^{(\gamma-1) / 2 \gamma}\right]}{A R(1-\gamma) \sqrt{\frac{\gamma T_{1}}{R}\left(\frac{2}{\gamma+1}\right)^{(\gamma+1) /(\gamma-1)}}}
$$

where $P_{1}$ is the propellant pressure upstream from the injector, and $\mathrm{P}_{2}$ is the pressure at the exit. The other variables are chamber volume (V), injection area (A), and gas constant (R) Thus, how long it will take for a propellant gas to occupy a given chamber volume depends, not only on the propellant $(\gamma, R)$, but on the size of the injector and the pressure drop across it. The estimated injection times for various propellant gases are plotted in Figures 3 through 6. As shown in Figs. 3 and 4, it takes a longer time for propane gas to "fill" a given volume, as compared with hydrogen gas, assuming the same injection conditions for both. For example, it takes $23 \mathrm{msec}$ for hydrogen to occupy a volume of $5.148 \times 10^{-4}$ $\mathrm{m}^{3}$, while propane takes $138 \mathrm{msec}$ at the same injection conditions (same injector, same $T_{1}, P_{1} / P_{2}$ ).

On the other hand, assuming that the propellants are injected into the detonation chamber as a pre-mixed, stoichiometric mixture, it is found that the time required ranges from 14 to $106 \mathrm{msec}$ for various mixtures to fill volumes between $5.148 \times 10^{-4}$ and $2.085 \times 10^{-3} \mathrm{~m}^{3}$ using a 0.50-inch diameter injector. If the injector diameter is doubled, the injection times for the same volumes are reduced to 3.57 and $26 \mathrm{msec}$, respectively.

Considering the very short injection times required by a high frequency PDE, it is estimated that only a fraction of the detonation chamber contains detonable mixture during each cycle. That is why it is so important to ensure that the propellant gases are well mixed and at the stoichiometric conditions for a given performance state at least in the vicinity of the ignition source. The location of the ignitor, thus becomes another design constraint for the PDE.

\subsection{Mixing Characterization}

To study the mixing characteristics inside a detonation chamber, jets of hydrogen and oxygen were simulated 
entering a two-dimensional chamber, using a computer code originally developed by $\mathrm{J}$. White in 1994, under contract with NASA Langley. The CFD code has built-in a number of diffusion, reaction, and flow models which makes it appropriate for this application. The computer program solves the Reynolds-averaged Navier-Stokes equations using several turbulence models. For this study, a $\kappa-\omega$ turbulence model was chosen. The two-dimensional computational domain matches the size of two detonation chambers, one chamber is $45.72 \mathrm{~cm}$ long and $7.62 \mathrm{~cm}$ wide, the second has the same length but is $5 \mathrm{~cm}$ wide. The computational mesh has $129 \times 129$ grid nodes. Front-wall and lateral-wall injection methods are studied. Front-wall injection is characterized by hydrogen and oxygen jets injected through the closed-end wall of the chamber at some angle with the chamber axis. With lateral-wall injection the fuel and oxidizer are injected transverse to the chamber axis, through opposite walls. A design that matches this side-wall configuration was reported in Reference 11. The injector's size was of the order of $0.635 \mathrm{~cm}$ (1/4 inch) in most cases. Multiple fuel and oxidizer injectors were also modeled to study the interaction that results from multiple-jets.

Injection pressure, temperature, and Mach number were assumed to be, in most cases, $1 \mathrm{~atm}, 300 \mathrm{~K}$, and unity, respectively. Due to the difference in molecular structure, the flow characteristics of hydrogen are different from those of oxygen. Therefore, in order to keep the fuel to oxidizer ratio within practical values, the injection conditions were chosen as those given in Table 2. Other injection angles and injection conditions have also been considered.

The velocity vector field in Fig. 7 illustrates the mixing of fuel and oxidizer jets injected through the closed-end wall of two combustion chambers. The upper two velocity fields correspond to jets of oxygen and hydrogen injected into the same chamber at different angles. The jet on the upper part of the domain is oxygen. Since the oxidizer and fuel jets have different velocities, a gross shear effect takes place and coherent structures develop throughout the mixing layers, enhancing the mixing. It is also observed that when the angle is increased from $20^{\circ}$ to $45^{\circ}$, the interaction of the fuel and oxidizer is stronger. However, downstream at a plane $10 \mathrm{~cm}$ from the closed-end wall of the chamber, the effect is reduced. In the second case, the mixing layer is thicker, indicating increased turbulence which pushes the gases faster against the opposite wall. The lower vector field results when the fuel and oxidizer jets are injected into a smaller diameter chamber. In this case, the lower jet is hydrogen, with an initial diameter of $0.47 \mathrm{~cm}$, injected at a $45^{\circ}$ angle, and the upper jet is oxygen, initial diameter of $0.71 \mathrm{~cm}$, injected at $26^{\circ}$. Selecting two different injection angles was done after observing that the resulting moment of impinging jets was directed "upwards", and it was theorized that, in order to get a resulting moment along the chamber axis, with a zero transverse component, the two jets having different densities, velocities, and masses would have to be injected at different angles. Two distinct effects are present in case c), the one due to the smaller gap between the injectors, and the one resulting from the different injection angles and different initial diameters. Thus, the jets interact differently as compared with the first two cases a) and b). The resulting vector field seems to have a more uniform gas core, but the mixing pattern observed in 
a) and b) is not present. In all cases, however, mixing is not perfect

The mixing characteristics of the hydrogen and oxygen jets can also be inferred from the gases mass distribution in the combustion chamber. Figure 8 shows contour plots of $\mathrm{O}_{2}$ mass fraction for various injection conditions. The shadow of the hydrogen jet is also included in these plots. As shown, the oxygen diffuses towards the open end of the chamber, where it tends to "fill the entire volume". However, in the vicinity of the closed end, the oxygen jet is moved upward by the effect of the hydrogen. jet and, within the first $10 \mathrm{~cm}$, the upper part of the chamber contains only oxidizer, and the lower part mostly fuel. This would imply that the gases in this region are not mixed to the stoichiometric proportions required for detonation. However, toward the exit plane of the chamber, a better mixture ratio is apparent.

The mixing characteristics in the chamber are quite different when the propellants are injected from opposing walls. The flowfield for this injection mode is shown in Fig. 9, where the velocity field is illustrated with vector and velocity contour plots, for cases 6 and 7, with same injection conditions but different chamber volume (please refer to Table 2). One distinctive feature of these flowfields is the relatively low axial velocity of the mixing layer, indicating a longer residence time for the propellants, which would allow them more time to mix at the molecular level. The vortices observed in the first case occur at the location where the hydrogen impinges with the oxygen jet, where the diffusion of reactants occurs. The species distributions for these two cases are shown in Fig. 10. The diameter of the chamber has an effect on the mixing characteristics of the fuel-oxidizer jets. Intuitively, one should expect that, with similar injection conditions, a smallerdiameter chamber would have better mixing characteristics than a larger one. This is because the jets penetrate farther into the opposite side, impinging upon each other with higher intensity.

The rate of mixing between the propellant jets can be influenced by many factors. The initial jet diameter, injection angle, velocity ratio $\mathrm{u}_{\mathrm{f}} / \mathrm{u}_{\mathrm{o}}$, density ratio, the turbulence properties of the jet, the presence of other jets, both adjacent and opposed, the proximity of walls, and the length of the jet path are some of the most important. In this paper only the effects of initial jet diameter and injection angle are included. In addition, three-dimensional effects are also possible, and modeling with a 2-D domain might shadow some features of the mixing process. Thus, more data are needed for optimizing the injection process in a given detonation chamber.

\section{CONCLUDNG REMARKS}

A study was made of the injection and mixing processes, aimed at providing a model to support pulsed detonation combustion research. Gaseous hydrogen and oxygen jets were simulated with a CFD code, injected into a two-dimensional chamber, at different injection conditions. The parametric study included effects of injection angle and jet initial diameter, keeping mixture ratios above stoichiometric values.

Front-end wall and lateral-wall injection schemes were modeled. The velocity field and the species mass distributions in the chamber provide a measure of the degree of mixing.

Injection and mixing of propellants in the combustion chamber of multi-cycle 
PDEs needs to be optimized. This study, although limited in scope, provides additional insight which can be helpful in the design of practical systems.

Work in progress ${ }^{18}$ include the development of a chemical kinetics model to simulate the combustion process with hydrocarbon-air and hydrocarbon-oxygen mixtures. It is expected that the study will yield numerical predictions of critical detonation parameters applicable to practical PDEs.

\section{REFERENCES}

1. Hinkey, J.B., Bussing, T.R.A., and Kaye, I., "Shock Tube experiments for the Development of a Hydrogen-Fueled Pulse Detonation Engine", AIAA 95-2578.

2. Bussing, T.R.A, Bratkovich, T.E., and Hinkey Jr., J.B., "Practical Implementation of Pulse Detonation Engines", AIAA 972748.

3. Stanley, S.B., Stuessy, W.S, and Wilson, D.R., "Experimental Investigation of Pulse Detonation Wave Phenomenon", AIAA 952197.

4. Stuessy, W.S. and Wilson, D.R., Influence of Nozzle Geometry on the Performance of a Pulse Detonation Engine", AIAA 97-2745.

5. W.S. Stuessy and D.R. Wilson, "Experimental Investigation of an Annular Multi-Cycle Pulsed Detonation Wave Engine", AIAA 97-0808.

6. Eidelman, S. and Grossmann, W., "Pulsed Detonation Engine Experimental and Theoretical Review", AIAA 92-3168.
7. Musielak, D.E., "Simulation of the Fluid Dynamic Behaviour of Transverse Hydrogen Injection in a Hypersonic Airstream", AIAA 97-2224.

8. J.L. Cambier and Tegner, J.K, "Strategies for PDE Performance Optimization", AIAA 97-2743.

9. I. Glassman, Combustion, Second Edition, Academic Press, 1987.

10. McBride, B. and Gordon, S., NASA Lewis Chemical Equilibrium Program CEA, Sept. 1997, NASA RP-1311, Part I (1994) and NASA RP-1311, Part II (1996).

11. P.P. Lo and D.E. Gonzalez, "Development of a Fuel Injection System for a High Frequency Pulse Detonation Engine", AIAA 97-2744.

12. Westbrook, C.K, Pitz, W.J, and Urtiew, P.A., "Chemical Kinetics of Propane Oxidation in Gaseous Detonations", 9th ICODERS, France, July 3-8, 1983.

13. Beeson, H.D., McClenagan, R.D, Bishop, C.V, et al., "Detonability of Hydrocarbon Fuels in Air", 1990.

14. Bull, D.C, "Towards an Understanding of the Detonability of Vapor Clouds", in Fuel-Air Explosions, University of Waterloo Press 1982, p. 139.

15.Westbrook, C.K., "Chemical Kinetics in Gaseous Detonations", in Fuel-Air Explosions, University of Waterloo Press 1982, p. 189.

16. Sichel, M., "The Detonation of Sprays: Recent Results", in Fuel-Air Explosions, Univ. of Waterloo Press, Nov. 4-6, 1981. 
17. Dabora, E.K., "Fundamental Mechanism of Liquid Spray Detonations", in Fuel-Air Explosions, University of Waterloo Press, Nov. 4-6, 1981, p. 245.
18. Musielak, D.E., "Computational Study of Detonation and Shock-Induced Combustion", (in preparation).

TABLE 1

Calculated* Values of the Physical Parameters in Stoichiometric Detonation Mixtures

\begin{tabular}{|c|c|c|c|c|}
\hline & & State 1 & State 2 & State 3 \\
\hline Mixture: & $2 \mathrm{H}_{2}+\mathrm{O}_{2}$ & & & \\
\hline$P(\mathrm{~atm})$ & & 1 & 32.3 & 18.788 \\
\hline $\mathrm{T}(\mathrm{K})$ & & 298.15 & 1898.91 & 3679.6 \\
\hline$\rho / \rho_{1}$ & & 1 & 5.07 & 1.8388 \\
\hline $\mathrm{u}(\mathrm{m} / \mathrm{s})$ & & 2836.9 & 559.25 & 1542.8 \\
\hline Mach Number & & 5.2744 & 0.412 & 1.0 \\
\hline Mixture: & $\mathrm{C}_{3} \mathrm{H}_{8}+5 \mathrm{O}_{2}$ & & & \\
\hline$P(\mathrm{~atm})$ & & 1 & 66.4 & 36.195 \\
\hline $\mathrm{T}(\mathrm{K})$ & & 298.15 & 2817.53 & 3825.6 \\
\hline$\rho / \rho_{1}$ & & 1 & 7.03 & 1.8574 \\
\hline $\mathrm{u}(\mathrm{m} / \mathrm{s})$ & & 2357.2 & 335.49 & 1269.1 \\
\hline Mach Number & & 7.6807 & 0.356 & 1.0 \\
\hline Mixture: & $\mathbf{H}_{2}+\mathbf{A i r}$ & & & \\
\hline P (atm) & & 1 & 27.11 & 15.598 \\
\hline $\mathrm{T}(\mathrm{K})$ & & 298.15 & 1639.31 & 2946.3 \\
\hline$\rho / \rho_{1}$ & & 1 & 4.93 & 1.8042 \\
\hline $\mathbf{u}(\mathrm{m} / \mathrm{s})$ & & 1965.8 & 398.71 & 1089.6 \\
\hline Mach Number & & 4.8341 & 0.418 & 1.0 \\
\hline Mixture: & $\mathrm{C}_{3} \mathbf{H}_{8}+\mathbf{A i r}$ & & & \\
\hline$P(a t m)$ & & 1 & 32.38 & 18.25 \\
\hline $\mathrm{T}(\mathrm{K})$ & & 298.15 & 1791.11 & 2822.4 \\
\hline$\rho / \rho_{1}$ & & 1 & 5.39 & 1.8106 \\
\hline $\mathrm{u}(\mathrm{m} / \mathrm{s})$ & & 1797.2 & 333.38 & 992.6 \\
\hline Mach Number & & 5.3067 & 0.402 & 1.0 \\
\hline
\end{tabular}

* CEA (Chemical Equilibrium) Program 

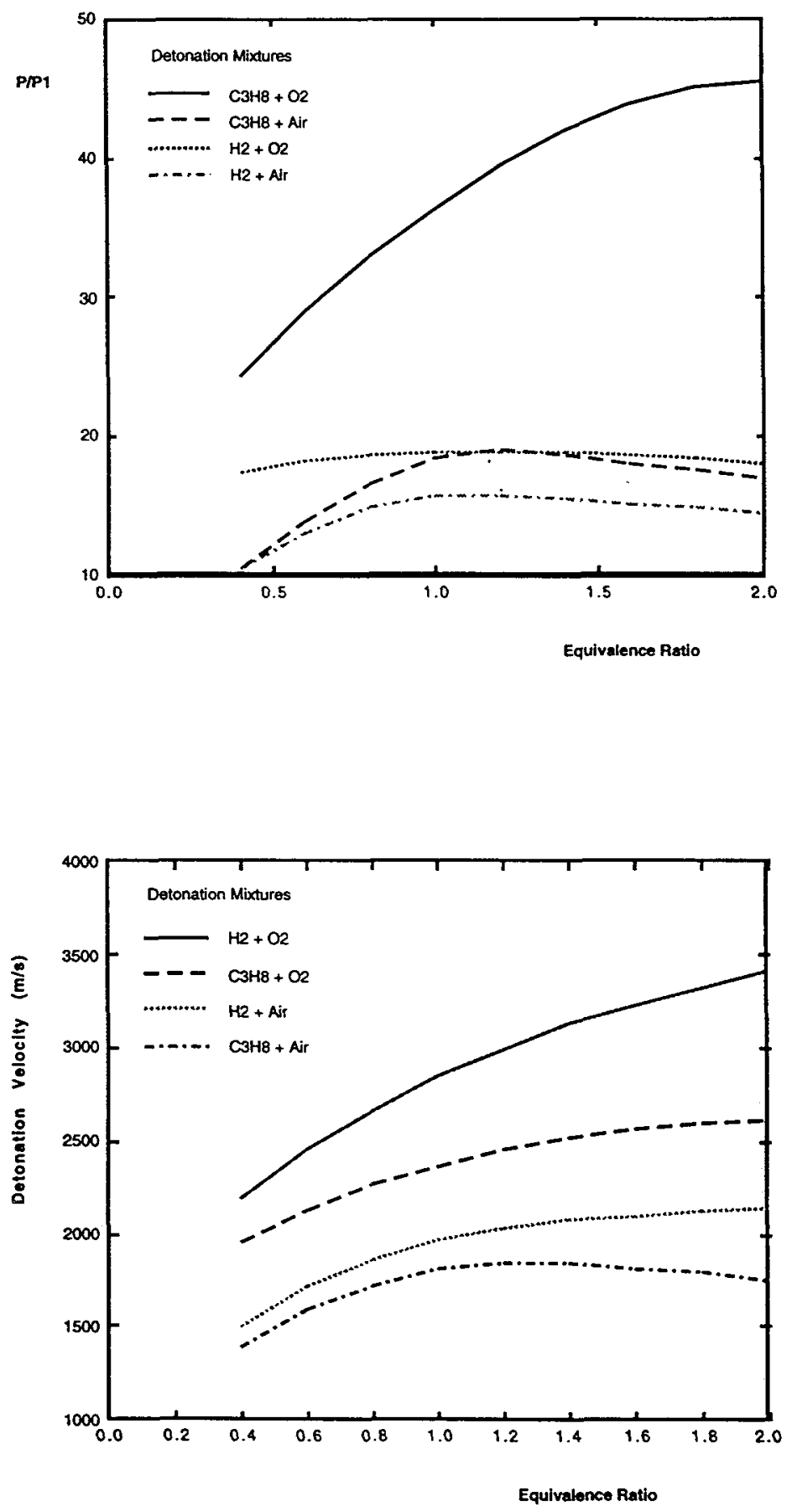

Fig. 1 Detonation Parameters Showing Sensitivity to Mixture Ratios 


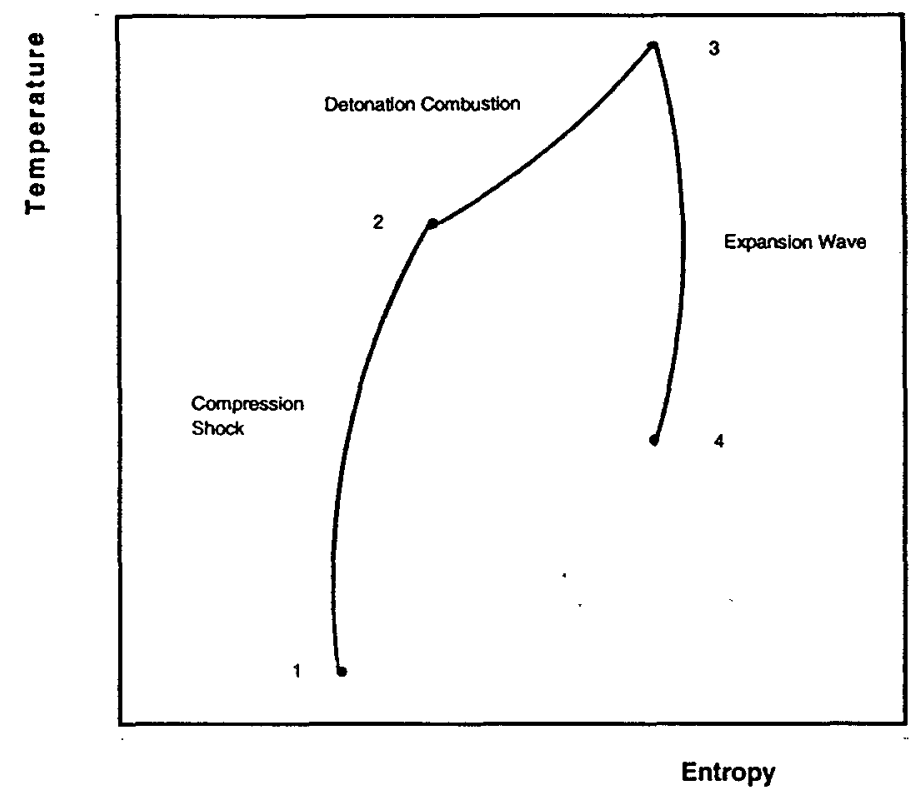

Fig. 2 Thermodynamic Cycle for Pulse Detonation Propulsion

TABLE 2

INJECTION CONDITIONS

\begin{tabular}{|c|c|c|c|c|c|c|}
\hline$\overline{\mathrm{CASE}}$ & $\begin{array}{l}\text { No. } \\
\text { Jets }\end{array}$ & $\begin{array}{l}\text { Injection } \\
\text { Angle }\end{array}$ & $\begin{array}{l}\text { Jet Size } \\
(\mathrm{mm})\end{array}$ & $\begin{array}{l}\text { Spacing } \\
\text { (cm) }\end{array}$ & V/A & $\begin{array}{l}\text { Mass Ratio } \\
\left(\mathrm{f}=\mathrm{m}_{\mathrm{f}} / \mathrm{m}_{\mathrm{o}}\right)\end{array}$ \\
\hline \multicolumn{7}{|c|}{ FRONT-END INJECTION: } \\
\hline case 1 & 2 & $20^{\circ}$ & 6.75 & 2.57 & 29.12 & 0.182 \\
\hline case2 & 2 & $45^{\circ}$ & 6.75 & 2.57 & 29.12 & 0.185 \\
\hline case 3 & 2 & $45^{\circ}$ & 6.75 & 2.57 & 29.12 & 0.253 \\
\hline case 4 & 2 & $45^{\circ}$ & 6.74 & 1.47 & 13.06 & 0.245 \\
\hline $\operatorname{case}^{\mathrm{a}, \mathrm{b}}$ & 2 & $45^{\circ} \& 26^{\circ}$ & 7.13 & 0.99 & 16.28 & 0.161 \\
\hline \multicolumn{7}{|c|}{ SIDE-WALL INJECTION: } \\
\hline $\operatorname{case}^{\mathrm{c}}$ & 4 & - & 5.33 & - & 27.09 & 0.205 \\
\hline $\operatorname{case} 7^{c}$ & 4 & - & 5.33 & - & 12.08 & 0.205 \\
\hline
\end{tabular}

a $45^{\circ}$ for fuel jet, $26^{\circ}$ for oxygen jet.

b Slot size for oxygen jet. Fuel injected through slightly smaller slot.

c Two slot sizes for both fuel and oxygen jets. 


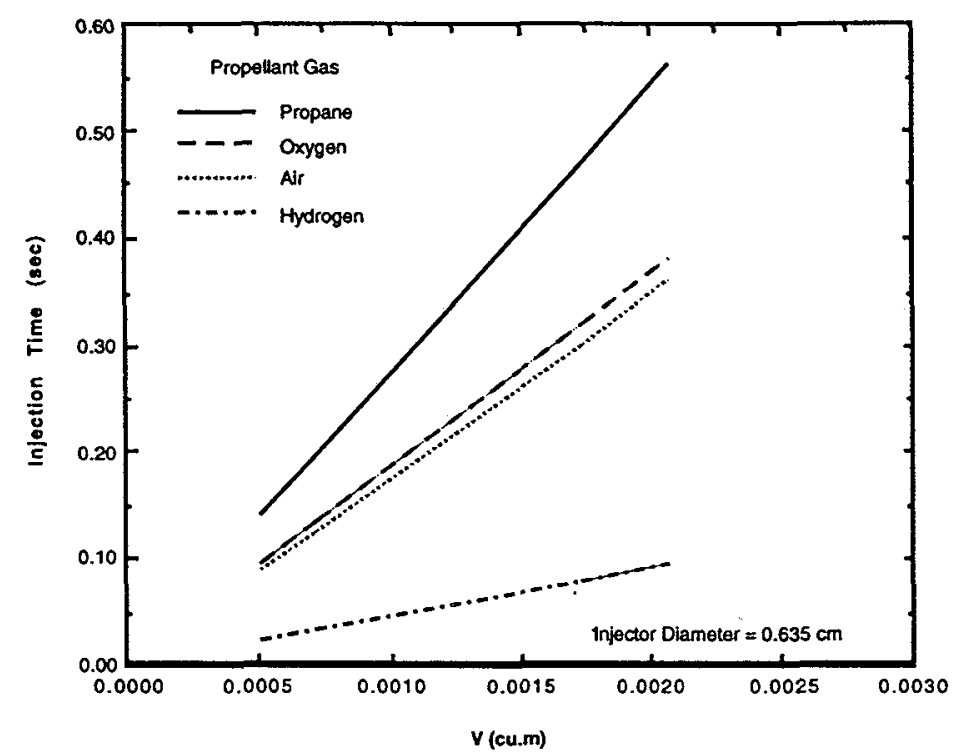

Fig. 3 Injection Time for Single-Gas Jet. Injection $\mathrm{P}_{2} / \mathrm{P}_{1}=4$, Diameter $=0.25$ "

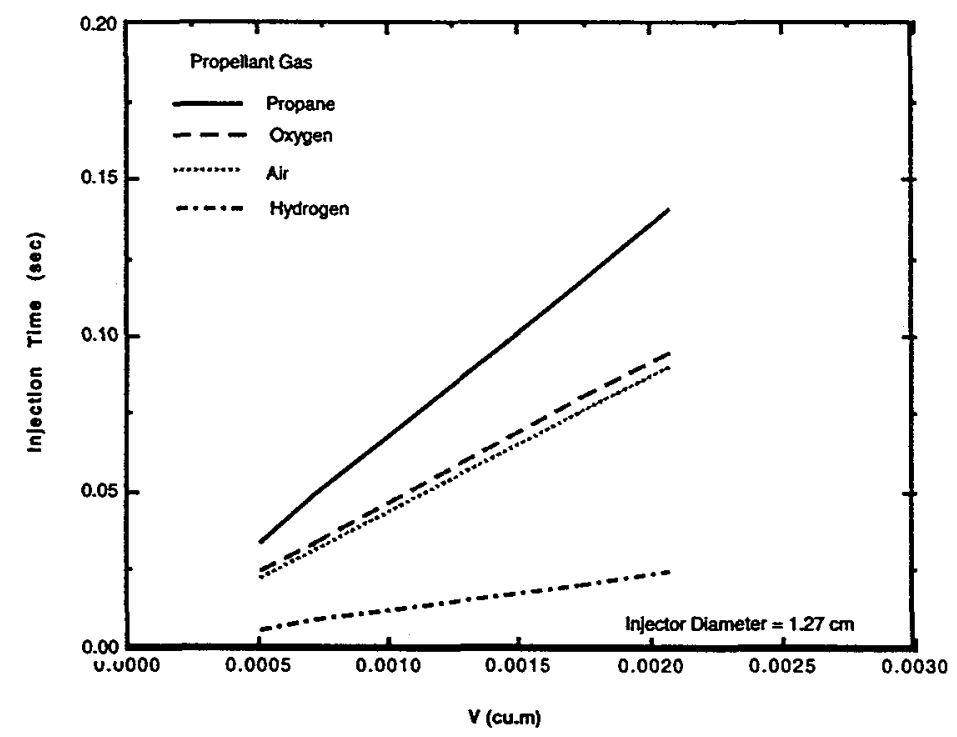

Fig. 4 Injection Time for Single-Gas Jet. Injection $\mathrm{P}_{2} / \mathrm{P}_{1}=4$, Diameter $=0.50$ " 


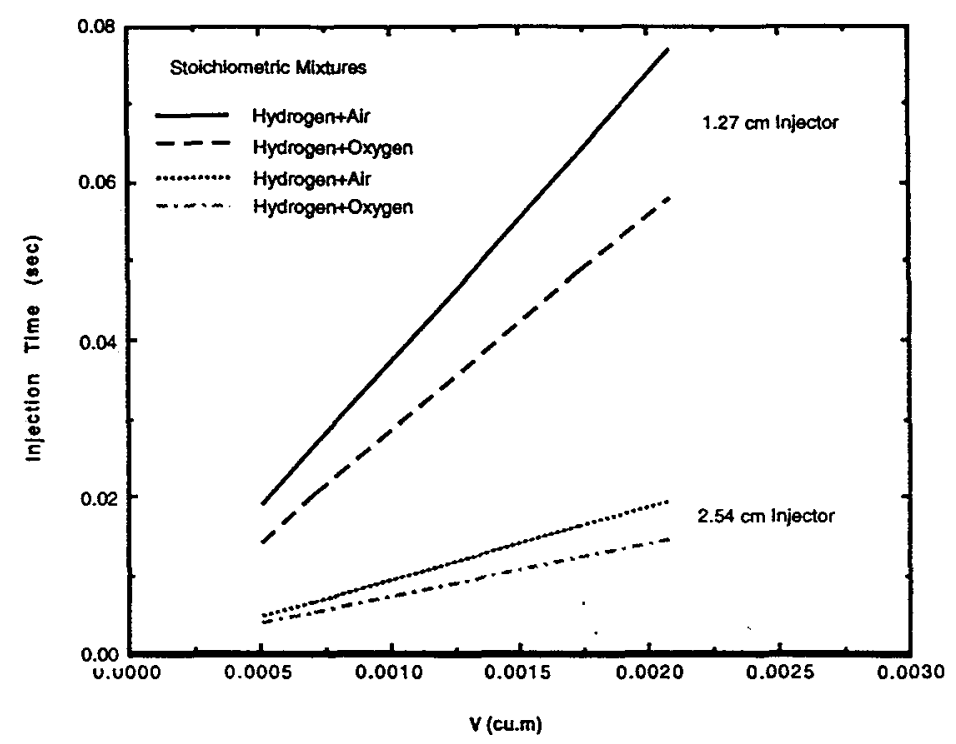

Fig. 5 Injection Time for Pre-Mixed Fuel/Oxidizer Jet. Injection $\mathrm{P}_{2} / \mathrm{P}_{1}=4$, Dia. $=0.50$ "

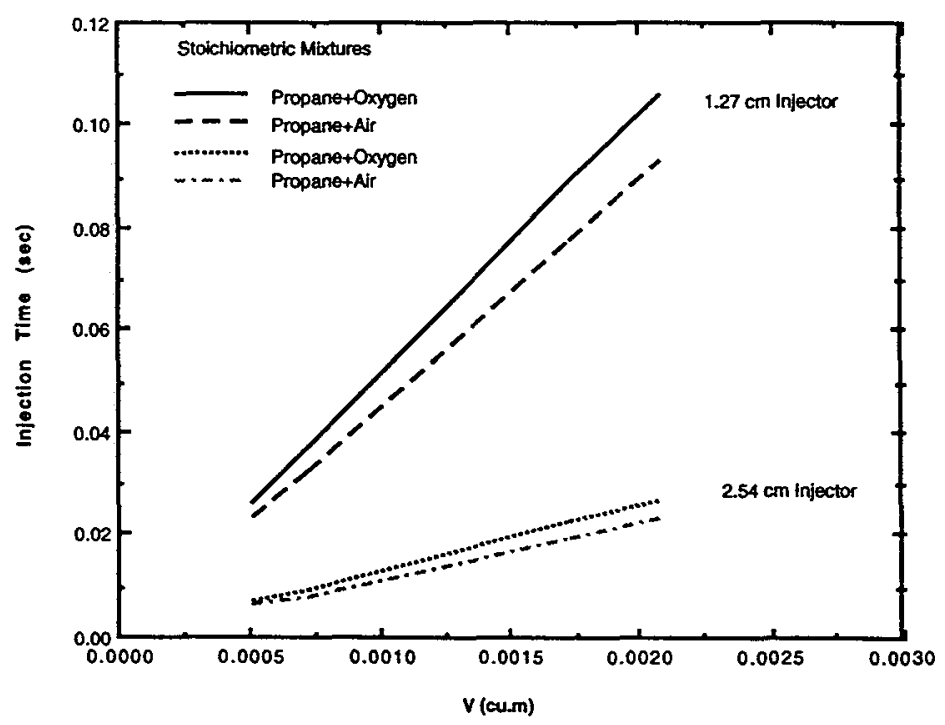

Fig. 6 Injection Time for Pre-Mixed Fuel/Oxidizer Jet. Injection $\mathrm{P}_{2} / \mathrm{P}_{1}=4$, Dia. $=1.0$ " 

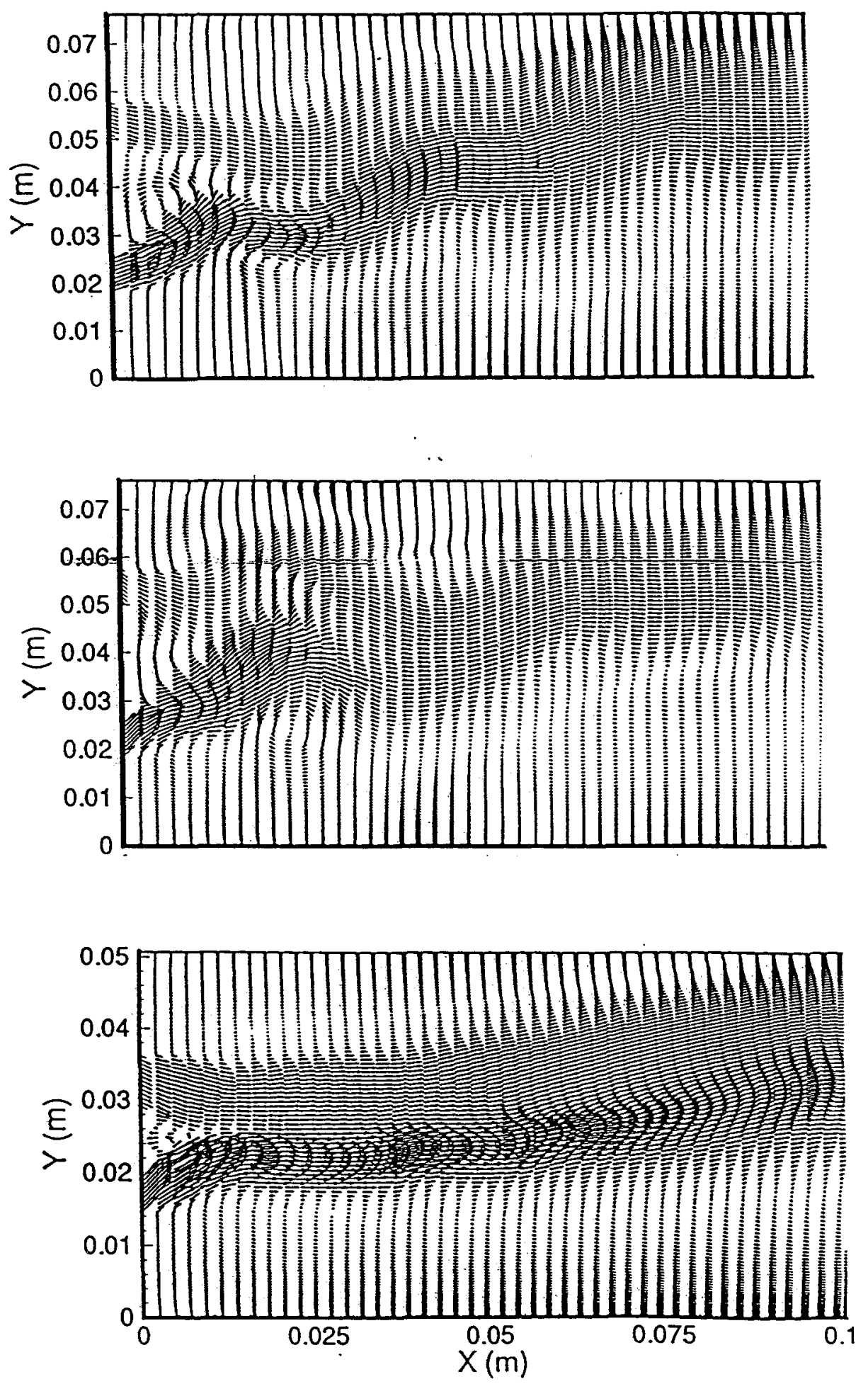

Fig. 7 Front-Wall Injection Velocity Field, a) V/A $=29, \alpha=20^{\circ}, \phi=1.44$, b) $\mathrm{V} / \mathrm{A}=29, \alpha=45^{\circ}, \phi=1.46$, c) $\mathrm{V} / \mathrm{A}=16, \alpha=45^{\circ}-26^{\circ}, \phi=1.27$ 
02 Mass Fraction
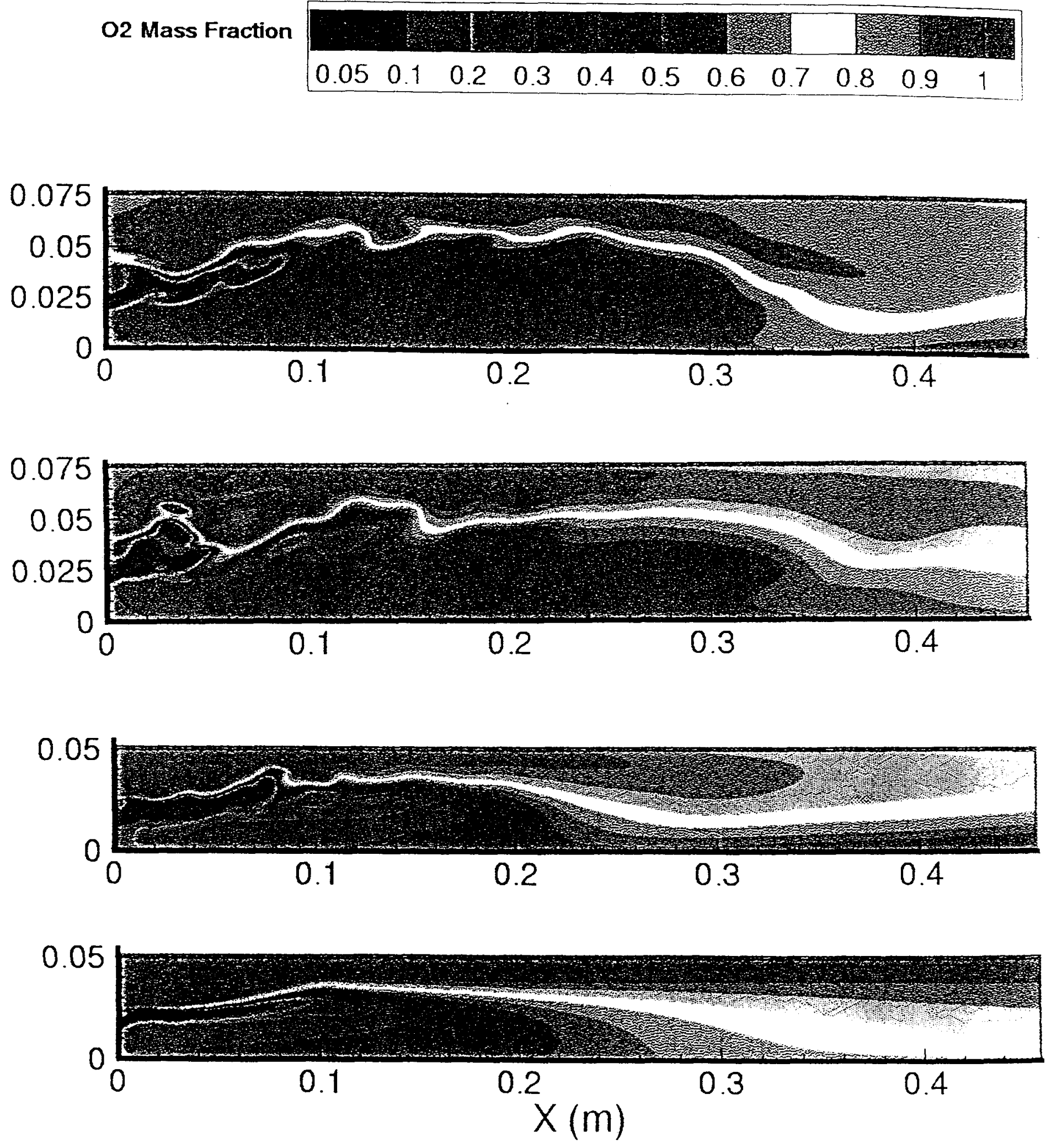

Fig. 8 Front-Wall Injection Hydrogen and Oxygen Mass Fraction Distribution,

a) $\mathrm{V} / \mathrm{A}=29, \alpha=20^{\circ}, \phi=1.44$, b) $\mathrm{V} / \mathrm{A}=29, \alpha=45^{\circ}, \phi=1.46$,

c) $\mathrm{V} / \mathrm{A}=13, \alpha=45^{\circ}, \phi=1.94$, d) $\mathrm{V} / \mathrm{A}=16, \alpha=45^{\circ}-26^{\circ}, \phi=1.27$ 


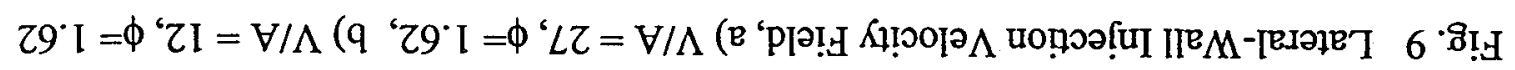
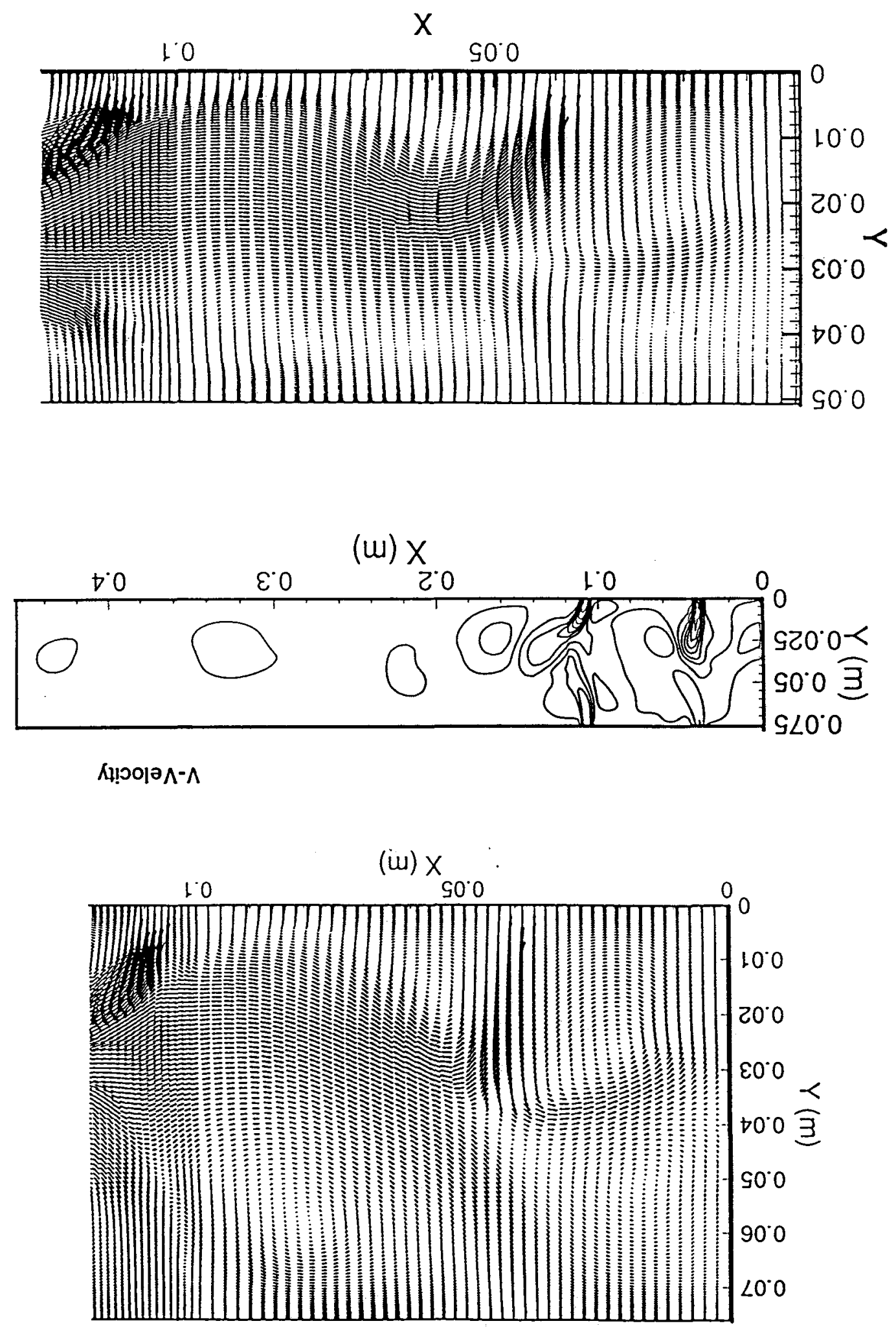

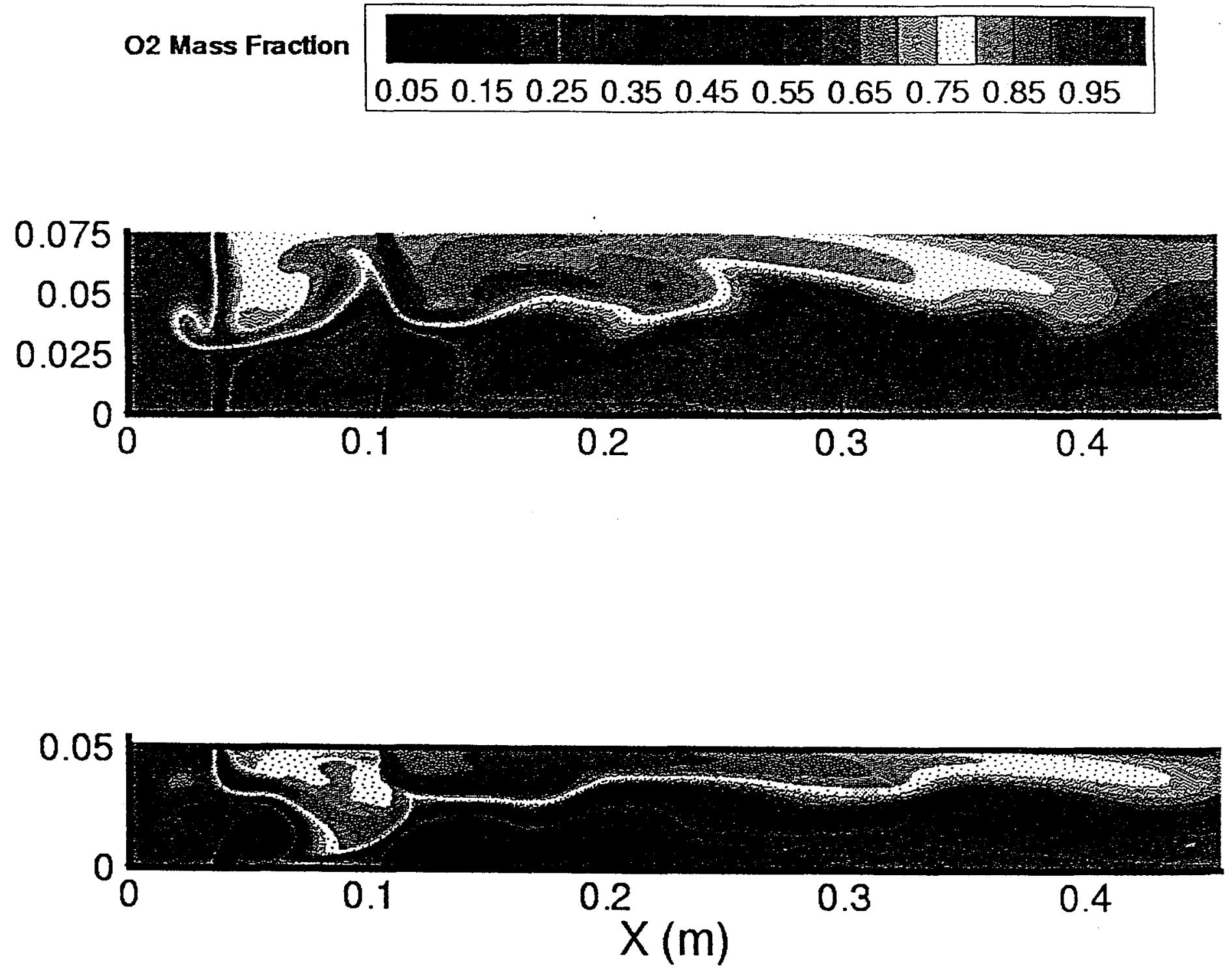

Fig. 10 Lateral-Wall Injection Hydrogen and Oxygen Mass Fraction Distribution,

a) $\mathrm{V} / \mathrm{A}=27, \phi=1.62$, b) $\mathrm{V} / \mathrm{A}=12, \phi=1.62$ 